\title{
Experimental and Analytical Study of Highly Tunable Electrostatically Actuated Resonant Beams
}

\author{
Amal Z. Hajjaj, Abdallah Ramini, and Mohammad I. Younis \\ Physical Sciences and Engineering Division, King Abdullah University of Science and Technology \\ Thuwal 23955-6900, Saudi Arabia \\ E-mail: mohammad.younis@kaust.edu.sa
}

\begin{abstract}
.
We demonstrate theoretically and experimentally highly tunable clamped-clamped microbeam resonators actuated with electrostatic forces. Theoretically, the Galerkin procedure is used to solve for the static deflection as well as the eigenvalue problem as a function of the DC voltage for different values of the ratio between the air gap and the thickness of the microbeam. We demonstrate theoretically and experimentally that the natural frequency of the microbeam can increase or decrease with the increase of the DC polarization voltage depending on the ratio between the air gap and the thickness. Hence, we show that unlike the classical softening effect of the DC voltage; by careful designs of the microbeams, the DC bias can be used to effectively increase the resonance frequencies by several factors. Experimental data are presented for two case studies of silicon beams showing effective increase of their fundamental resonance frequencies by more than 50-80\%. Excellent agreement is reported among the theoretical and experimental results.
\end{abstract}

\section{Introduction}

Tunability of microelectromechanical (MEMS) resonators is highly desirable feature for various applications, including communications [1], filtering [2], gyroscopes [3], energy harvesting [4], and signal processing [5]. Tunability refers to the ability to control or tune the resonance frequency, linear natural frequency, of a resonator by increasing or decreasing it to the desirable value. Resonators can be bulk-mode, in which a thick block of microstructure is excited through wave propagation; or it can be based on thin-film technology, in which a thin compliant microstructure is driven into bending-mode vibrations. This paper is concerned with the second category. One of the extensively used methods to excite these resonators is the electrostatic actuation. In this work, we study electrostatically actuated microbeams, which represent a main component of this class of resonators. It is composed of an elastic clamped-clamped straight microbeam hanging above a stationary electrode. A DC voltage and an AC component to generate harmonic motions are applied on the microbeam.

Classically, the effect of the DC bias on microstructures has been considered mainly to decrease their resonance frequencies through the softening effect [6]. On the other hand, at the Nano scale, there have been experimental evidences demonstrating the tunability, mainly the increase of the resonance frequencies of Carbon Nano Tube CNT resonators when increasing the DC gate voltage [7]. This behavior has been attributed to the high stiffness of CNTs, and hence, was thought to be a special feature of these small structures [8]. Tajaddodianfar et al [9] investigated using the strain gradient theory the tunability of size-dependent nano-arches actuated electrostatically. Kosinsky et al [10] demonstrated experimentally the ability to increase and decrease the resonance frequency of nanomechanical resonators electrostatically. As will be demonstrated in this work, such a behavior can be actually exploited at the micro scale as well with a proper design of certain parameters of the resonators. Several studies have 
investigated the effect of the size-dependent mico and nano beams electrostatically actuated on the limit buckling load and the pull-in voltage [11-12].

Many approaches have been investigated in the literature to tune the resonance behavior of MEMS resonators. Remtema and Lin [13] investigated experimentally and theoretically the integration of a microbeam that can be resistively heated to generate thermal stress on a comb-shape to tune the frequency. They showed that the resonance frequency could be reduced by $6.5 \%$. Yamamoto et al [14] showed that the resonance frequency of superconducting resonators could be tuned by inserting a superconducting quantum interference device in the resonator and adjusted with a magnetic field. Controlling the DC voltage for electrostatically actuated microbeams is the more known approach since the early work of Nathanson [15]. However, it has been mainly used for decreasing the resonance frequency, which is also limited by the pull-in instability. Indeed, applying a DC voltage forces the microbeam to deflect towards the stationary electrode and then the beam collapses for relatively high value of DC voltage [16].

Several numerical techniques have been presented to describe the response of a microbeam under DC voltages, such as the Rayleigh-Ritz method [17], the reduced order method (ROM) [6], the finite element method (FEM) [18], the shooting method [19], and the quadratic differential method (DQM) [20].

Numerous studies have investigated numerically and experimentally the variation of the natural frequencies of clamped-clamped microbeams under electrostatic actuation [21-24]. Nayfeh et al [6] compared the normalized fundamental natural frequency calculated using the ROM with results obtained by solving the eigenvalue problem of the distributed-parameter system under DC loading using a shooting method presented by Abdel-Rahman et al [19] and the experimental results obtained by Tilmans et al [17]. They showed that the electrostatic force, as with the case of other capacitive structures, loweres the fundamental frequency of the clamped-clamped microbeam up to zero near pull-in.

On the other hand, few studies showed, using numerical methods, such as ROM, shooting, and FEM that the natural frequency might be shifted to higher values if the effect of the mid-plane stretching is taken into consideration in the model and for relatively high values of the ratio between the air gap and the thickness of the microbeam. This possibility was first demonstrated by Abdel-Rahman et al [19] who used a shooting method. They showed that the natural frequency shifted for higher values as increasing the DC polarization voltage before reaching the pull-in instability for high ratio between the air gap and the thickness of the microbeam. Batra et al [18] obtained similar results as that in [19] using FEM and analytical methods accounting for electrostatic fringing fields.

We note from the aforementioned review the lack of a rigorous study that sheds light on the reason behind the unexpected increase of the resonance frequency of resonators with the DC bias. Few theoretical studies have been presented at the micro scale to explain the increase of the fundamental frequency of the electrostatically actuated microbeam for high values of the ratio between the air gap and the thickness of the microbeam; however with no experimental data that demonstrate the phenomenon.

Our aim in this paper is to investigate theoretically and experimentally the effect of the ratio between the air gaps and the thickness of the microbeam on the natural frequency as changing the DC loading. We explain the conditions to tune the natural frequency of electrostatically actuated resonators by more than $100 \%$.

The rest of the paper is organized as follows. The problem model is derived in Section 2. The static response is analyzed in Section 3. The static results are used to solve the eigenvalue problem for different values of the ratio between the air gap and the thickness of the microbeam in Section 4. The experimental setup and case studies are presented in Section 5. The experimental validation is conducted in Section 6. Finally, the main conclusions are summarized in Section 7. 


\section{Problem formulation}

The device under consideration, Figure1, consists of a clamped-clamped microbeam actuated by a DC polarization voltage $V_{D C}$ and an AC harmonic voltage of amplitude $V_{A C}$ and of frequency $\hat{\Omega}$, and is subjected to a viscous damping of coefficient $\hat{c}$.

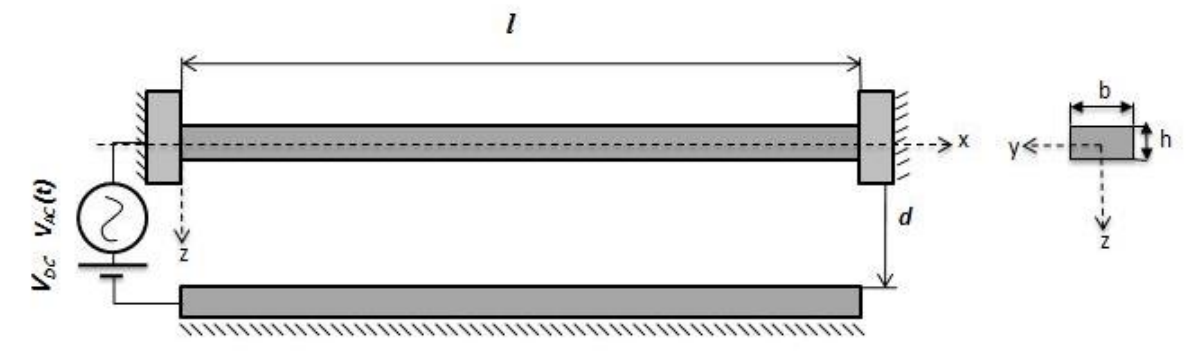

Figure 1: Schematic of an electrostatically actuated clamped-clamped microbeam.

The equation of motion governing the transverse deflection of the beam $\hat{w}(\hat{x}, \hat{t})$ is written as follows:

$$
\rho b h \frac{\partial^{2} \hat{w}}{\partial \hat{t}^{2}}+\hat{c} \frac{\partial \hat{w}}{\partial \hat{t}}+E I \frac{\partial^{4} \hat{w}}{\partial \hat{x}^{4}}=\left[\hat{N}+\frac{E A}{2 l} \int_{0}^{l}\left(\frac{\partial \hat{w}}{\partial \hat{x}}\right)^{2} d \hat{x}\right] \frac{\partial^{2} \hat{w}}{\partial \hat{x}^{2}}+\frac{1}{2} \varepsilon b \frac{\left(V_{D C}+V_{A C} \cos (\hat{\Omega} t)\right)^{2}}{(d-\hat{w})^{2}}
$$

where $\hat{x}$ is the position along the microbeam and $\hat{t}$ is time. This in-plane microbeam is of length $l$, of width $b$ and of thickness $h$. It has Young's modulus $E$ and material density $\rho$ and is assumed to have a rectangular cross section area $A=b h$ and a moment of inertia $I=b h^{3} / 12$. In this work, $\hat{N}$ presents the residual axial load. This microbeam is subjected to electrostatic force where $d$ is the gap width between the microbeam and the stationary electrode, and $\varepsilon$ is the dielectric constant of the medium. The microbeam is subjected to the following boundary conditions:

$$
\hat{w}(0, \hat{t})=\hat{w}(l, \hat{t})=0 \text { and }\left.\frac{d \hat{w}}{d \hat{x}}\right|_{(0, \hat{t})}=\left.\frac{d \hat{w}}{d \hat{x}}\right|_{(l, \hat{t})}=0
$$

For convenience, we introduce the nondimensional variables as below:

$$
w=\frac{\hat{w}}{d} ; x=\frac{\hat{x}}{l} \text { and } t=\frac{\hat{t}}{T}
$$

Where $T=\sqrt{\frac{\rho b h l^{4}}{E I}}$ is the time scale chosen for convenience. Substituting equation (3) into equations (1) and (2), we obtain the nondimensional equation of motion of the beam:

$$
\frac{\partial^{2} w}{\partial t^{2}}+c \frac{\partial w}{\partial t}+\frac{\partial^{4} w}{\partial x^{4}}=\left[N+\alpha_{1} \int_{0}^{1}\left(\frac{\partial w}{\partial x}\right)^{2} d x\right] \frac{\partial^{2} w}{\partial x^{2}}+\alpha_{2} \frac{\left(V_{D C}+V_{A C} \cos (\Omega t)\right)^{2}}{(1-w)^{2}}
$$

Subjected to the nondimensional boundary conditions:

$$
w(0, t)=w(1, t)=0 ;\left.\frac{\partial w}{\partial x}\right|_{(0, t)}=\left.\frac{\partial w}{\partial x}\right|_{(1, t)}=0
$$

The nondimensional parameters appearing in equation (4) are defined as below: 


$$
\alpha_{1}=6\left(\frac{d}{h}\right)^{2} ; \alpha_{2}=\frac{6 \varepsilon l^{4}}{E h^{3} d^{3}} ; N=\frac{l^{2}}{E I} \hat{N} ; c=\frac{l^{4}}{E I T} \hat{c} \text { and } \Omega=T \hat{\Omega}
$$

The deflection of the microbeam under the electrostatic force is split into a static deflection $w_{s}(x)$ due the DC component and a small dynamic deflection $w_{d}(x, t)$

$$
w(x, t)=w_{s}(x)+w_{d}(x, t)
$$

To determine the static deflection $w_{s}(x)$ we set the time derivatives as well as the AC force equal to zero:

$$
\frac{\partial^{4} w_{s}}{\partial x^{4}}=\left[N+\alpha_{1} \int_{0}^{1}\left(\frac{\partial w_{s}}{\partial x}\right)^{2} d x\right] \frac{\partial^{2} w_{s}}{\partial x^{2}}+\alpha_{2} \frac{V_{D C}{ }^{2}}{\left(1-w_{s}\right)^{2}}
$$

With the associated boundary conditions

$$
w_{s}(0)=w_{s}(1)=0 \text { and }\left.\frac{d w_{s}}{d x}\right|_{x=0}=\left.\frac{d w_{s}}{d x}\right|_{x=1}=0
$$

The linearized equation of motion describing the small dynamic behavior of the microbeam around the deflected shape is derived by substituting equation (7) into equation (4) and dropping the terms representing the equilibrium position, equations (8), and the nonlinear terms. This yields

$$
\frac{\partial^{2} w_{d}}{\partial t^{2}}+\frac{\partial^{4} w_{d}}{\partial x^{4}}=\left[N+\alpha_{1} \int_{0}^{1}\left(\frac{\partial w_{s}}{\partial x}\right)^{2} d x\right] \frac{\partial^{2} w_{d}}{\partial x^{2}}+\left[2 \alpha_{1} \int_{0}^{1} \frac{\partial w_{s}}{\partial x} \frac{\partial w_{d}}{\partial x} d x\right] \frac{\partial^{2} w s}{\partial x^{2}}+\frac{2 \alpha_{2}\left(V_{D C}+V_{A C} \cos (\Omega t)\right)^{2}}{\left(1-w_{s}\right)^{3}} w_{d}
$$

With the associated boundary conditions:

$$
w_{d}(0, t)=w_{d}(1, t)=0 \text { and }\left.\frac{\partial w_{d}}{\partial x}\right|_{x=0, t}=\left.\frac{\partial w_{d}}{\partial x}\right|_{x=1, t}=0
$$

\section{Static response}

Equations (8) and (9) describe, respectively, the static deflection of the microbeam and its associated boundary conditions. To solve equation (8), we refer to the Galerkin procedure in which we use the undamped linear mode shapes of a straight microbeam as basis functions $[6,21,26]$. Therefore the deflection is expressed as follows:

$$
w_{s}(x)=\sum_{0}^{n} u_{i} \phi_{i}(x)
$$

Where $u_{i}(i=0,1,2 \ldots n)$ denotes the nondimensional modal coordinates and ${ }_{i}(x)(i=0,1,2 \ldots n)$ denotes the undamped mode shapes of the straight unactuated beam governed by

$$
\frac{d^{4} \phi_{i}}{d x^{4}}-N \phi_{i}^{\prime \prime}-\omega_{i}^{2} \phi_{i}=0
$$

With the associated boundary conditions:

$$
\phi_{i}(0)=\phi_{i}(1)=0 \text { and }\left.\frac{d \phi_{i}}{d x}\right|_{x=0}=\left.\frac{d \phi_{i}}{d x}\right|_{x=1}=0
$$

Where $\omega_{\mathrm{i}}$ represents the $i^{\text {th }}$ natural frequency of the microbeam. Using five symmetric modes and assuming $N=0$, we solve equation (8) using the computational software program Mathematica for a range of DC voltage from zero to pull-in for different values of the ratio between the air gap and the thickness of the beam. This ratio is represented by the nondimensional parameter $\alpha_{1}$. The geometrical and 
mechanical parameters adopted for the simulations are as shown in Table 1:

\begin{tabular}{|l|l|}
\hline Parameters & Values \\
\hline Length $l$ & $600[\mu \mathrm{m}]$ \\
\hline Thickness $(h)$ & $2[\mu \mathrm{m}]$ \\
\hline Width $(b)$ & $25[\mu \mathrm{m}]$ \\
\hline Gap $(d)$ & $1-10[\mu \mathrm{m}]$ \\
\hline Young's Modulus $(E)$ & $154[\mathrm{GPa}]$ \\
\hline Density $(\rho)$ & $2332\left[\mathrm{~kg} / \mathrm{m}^{3}\right]$ \\
\hline
\end{tabular}

Table 1: Geometrical and mechanical properties of the microbeam.

In figure 2, we plot the maximum nondimensional static deflection $W_{\max }=w_{s}(0.5)$ as a function of the DC voltage for different values of the gap $d$. The maximum deflection increases as the electrostatic force presented by the DC voltage increases. We found that the maximum deflection decreases for high values of $\alpha_{1}$ at the same level of DC voltage. On the other hand, the pull-in instability is shifted for higher values of DC voltage as $\alpha_{1}$ increases. The reason for this is that as $\alpha_{l}$ increases by increasing $d$ for a fixed value of thickness $h$, the electrostatic force effect is weaken with respect to mid-plane stretching. This electrostatic effect is presented by $\alpha_{2} V_{D c}{ }^{2}$ [19]. As reported by Younis and coworkers in [19,21], Tilmans et al [17], and Hung et al [25] mid-plane stretching is crucial in the model to avoid underestimation of the pull-in instability limits.

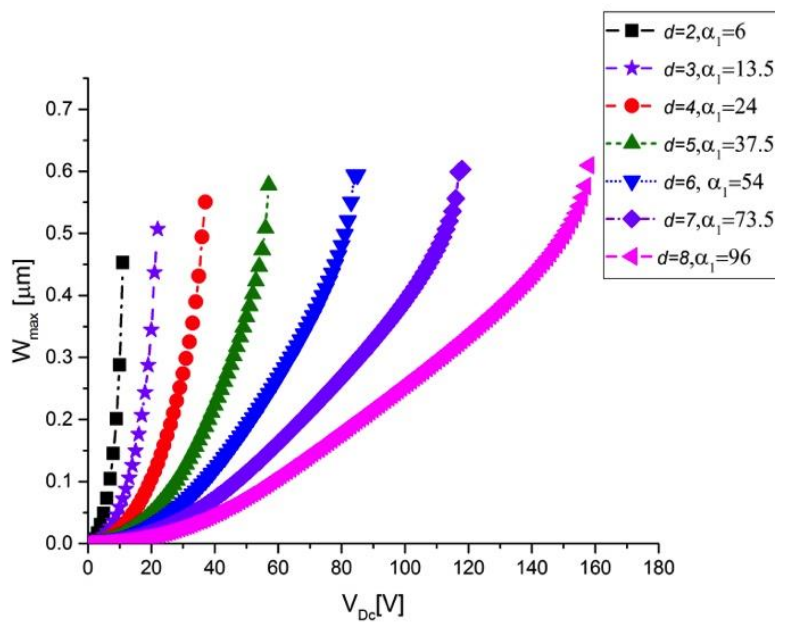

Figure 2: The maximum static defection of the microbeam versus the DC polorization voltage for diffecrent values of $\alpha_{1}$.

\section{The Natural frequency under the DC polarization voltage}

To determine the variation of the natural frequency of the microbeam under the DC polarization voltage, we resort to the Galerkin discretization to represent the dynamic deflection $w_{d}(x, t)$ and to solve the eigenvalue problem [26]. Toward this, we let:

$$
w_{d}(x, t)=\sum_{i=0}^{n} u_{i}(t) \phi_{i}(x)
$$


Where $u_{i}(t)(i=0,1,2 . . n)$ denotes the nondimensional modal coordinates and $\phi_{i}(x)(i=0,1,2 . . n)$ denotes the undamped mode shapes of the straight unactuated beam.

Then, we substitute equation (15) into equation (10), multiplying the outcome by the mode shape $j$, applying the orthogonality condition of the mode shapes, and integrating over the beam domain (from 0 to 1), which yields the below equation [26]:

$$
\ddot{u}_{j}+u_{j} \omega_{n o n, j}^{2}=\left[2 \alpha_{1} \int_{0}^{1} \phi_{j} \frac{\partial^{2} w_{s}}{\partial x^{2}} d x \int_{0}^{1}\left(\sum_{i=0}^{n} u_{i} \phi_{i} \frac{\partial w_{s}}{\partial x}\right) d x\right]+\left[\alpha_{1} \int_{0}^{1}\left(\frac{\partial w_{s}}{\partial x}\right)^{2} d x\right] \int_{0}^{1} \phi_{j}\left(\sum_{i=0}^{n} u_{i} \phi_{i}{ }^{\prime \prime}\right) d x+\int_{0}^{1} \phi_{j} \frac{2 \alpha_{2} V_{D C}^{2}}{\left(1-w_{s}\right)^{3}}\left(\sum_{i=0}^{n} u_{i} \phi_{i}\right) d x
$$

Using three symmetric mode shapes, the above system can be expanded into the following three modal equations:

$$
\begin{aligned}
& \ddot{u}_{1}+u_{1} \omega_{n o n, 1}^{2}=\left[2 \alpha_{1} \int_{0}^{1} \phi_{1} \frac{\partial^{2} w_{s}}{\partial x^{2}} d x \int_{0}^{1}\left(\sum_{i=0}^{3} u_{i} \phi_{i} \frac{\partial w_{s}}{\partial x}\right) d x\right]+\left[\alpha_{1} \int_{0}^{1}\left(\frac{\partial w_{s}}{\partial x}\right)^{2} d x\right] \int_{0}^{1} \phi_{1}\left(\sum_{i=0}^{3} u_{i} \phi_{i}{ }^{\prime \prime}\right) d x+\int_{0}^{1} \phi_{1} \frac{2 \alpha_{2} V_{D C}^{2}}{\left(1-w_{s}\right)^{3}}\left(\sum_{i=0}^{3} u_{i} \phi_{i}\right) d x \\
& \ddot{u}_{2}+u_{2} \omega_{n o n, j}^{2}=\left[2 \alpha_{1} \int_{0}^{1} \phi_{2} \frac{\partial^{2} w_{s}}{\partial x^{2}} d x \int_{0}^{1}\left(\sum_{i=0}^{3} u_{i} \phi_{i} \frac{\partial w_{s}}{\partial x}\right) d x\right]+\left[\alpha_{1} \int_{0}^{1}\left(\frac{\partial w_{s}}{\partial x}\right)^{2} d x\right] \int_{0}^{1} \phi_{2}\left(\sum_{i=0}^{3} u_{i} \phi_{i}^{\prime \prime}\right) d x+\int_{0}^{1} \phi_{2} \frac{2 \alpha_{2} V_{D C}^{2}}{\left(1-w_{s}\right)^{3}}\left(\sum_{i=0}^{3} u_{i} \phi_{i}\right) d x \\
& \ddot{u}_{3}+u_{3} \omega_{\text {non }, j}^{2}=\left[2 \alpha_{1} \int_{0}^{1} \phi_{3} \frac{\partial^{2} w_{s}}{\partial x^{2}} d x \int_{0}^{1}\left(\sum_{i=0}^{3} u_{i} \phi_{i} \frac{\partial w_{s}}{\partial x}\right) d x\right]+\left[\alpha_{1} \int_{0}^{1}\left(\frac{\partial w_{s}}{\partial x}\right)^{2} d x\right] \int_{0}^{1} \phi_{3}\left(\sum_{i=0}^{3} u_{i} \phi_{i}^{\prime \prime}\right) d x+\int_{0}^{1} \phi_{3} \frac{2 \alpha_{2} V_{D C}^{2}}{\left(1-w_{s}\right)^{3}}\left(\sum_{i=0}^{3} u_{i} \phi_{i}\right) d x
\end{aligned}
$$

Equations (17), (18) and (19) represent a discretized system of three ordinary differential equations. For a given $V_{D C}$, we compute the Jacobian of the system of equations (17), (18) and (19) and find the corresponding eigenvalues. Then, by taking the square root of these eigenvalues, we find the natural frequencies of the resonators under a DC voltage. A comparison of the normalized natural frequency calculated using the ROM to one obtained by shooting method [19] and experimentally [17] is shown by Nayfah et al [6] to validate this ROM procedure. In figure 3, we show the variation of the fundamental frequency as a function of the DC loading for different values of $\alpha_{1}$ for the same parameters used in Section 3. As demonstrated in Section 3, the pull-in instability shifts for higher values as $\alpha_{1}$ is increased. We demonstrate in figures $3 \mathrm{a}$ that the variation of the first natural frequency is highly dependent to the variation of the electrostatic force as well as the variation of the ratio between the air gap and the thickness of the microbeam.

For low values of $\alpha_{1}$ that are represented in figure $3 \mathrm{~b}$ by gaps comparable to the thickness of the microbeam, the fundamental frequency decreases as the DC voltage is increased, as traditionally known in the literature. In this case the effect of mid-plane stretching is negligible. However, for low DC voltage, regardless of the value of $\alpha_{1}$, the effect of the electrostatic force becomes dominant, which leads to the decrease of the natural frequency. For $20 \leq \alpha_{1} \leq 30$, the effect of the mid-plane stretching is comparable to the effect of the electrostatic force. Then, the natural frequency increases slightly with the increase of the DC voltage as shown in figure 3c. For $\alpha_{1} \geq 30$, figure 3d, the effect of the mid-plane stretching dominates the effect of the electrostatic force. As sweeping the DC voltage in this case, the natural frequency reaches more than $100 \%$ of increase with respect to its zero-voltage value. For example, for $\alpha_{I}=150$, the natural frequency can increase from $40 \mathrm{kHz}$ for $V_{D C}=0 \mathrm{~V}$ to $60 \mathrm{kHz}$ at $V_{D C}=100 \mathrm{~V}, 80 \mathrm{kHz}$ at $V_{D C}=160 \mathrm{~V}$ and $96 \mathrm{kHz}$ at $V_{D C}=220 \mathrm{~V}$. Therefore, we prove here that one can increase the frequency to more than twice its original value by increasing the gap between the microbeam and the stationary electrode and by increasing the DC voltage that is far from the pull-in instability. 


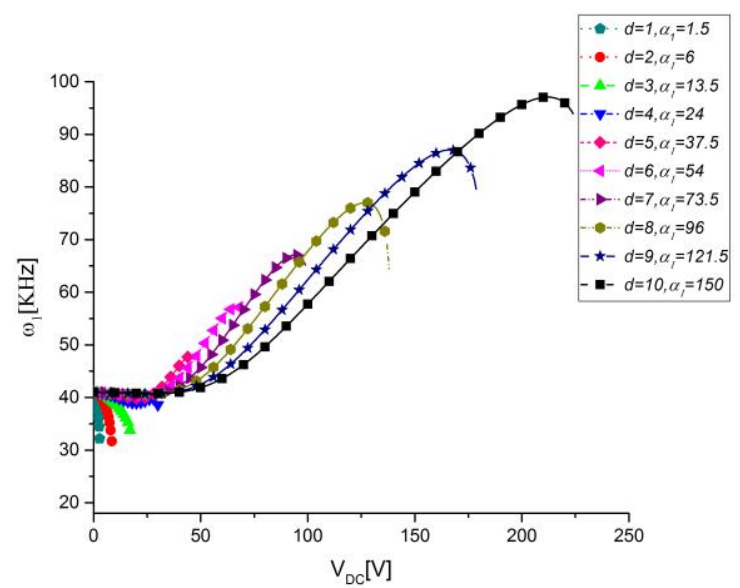

(a)

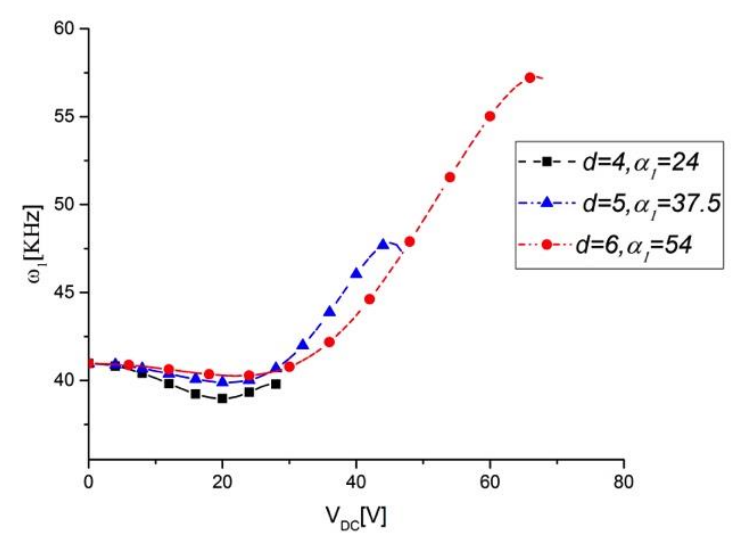

(c)

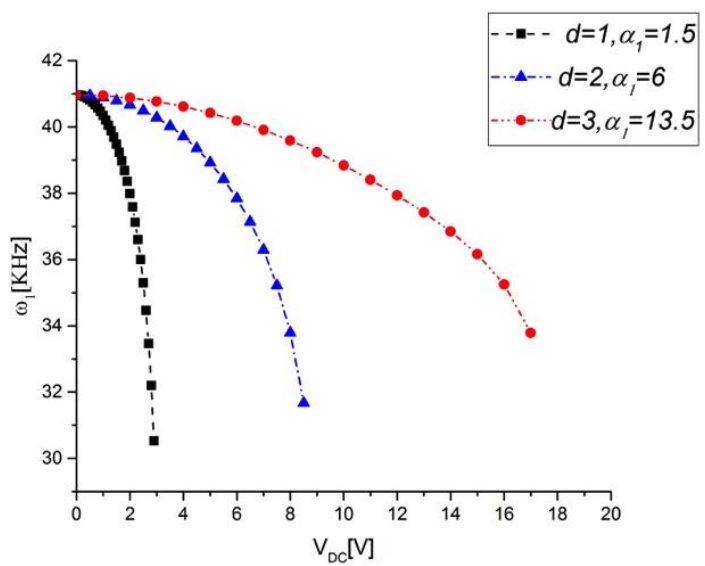

(b)

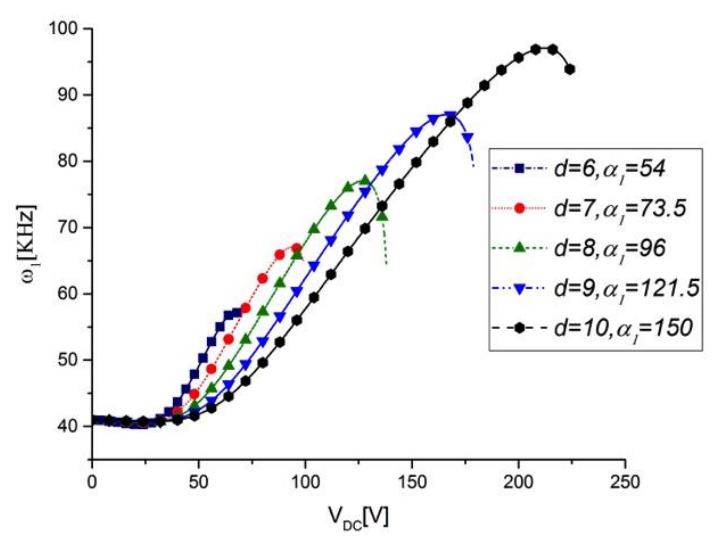

(d)

Figure 3: (a) The fundamental natural frequency versus the DC polarization voltage for different values of $\alpha_{1}$, (b), (c) and (d) represent enlarged view of (a) for low, medium and high values of $\alpha_{1}$, respectively. In the legends, $d$ has the unit of micro meters.

Figure 4 shows the variation of the fundamental frequency as a function of DC voltage for various values of nondimensional axial load for different values of $\alpha_{1}$. For low values of $\alpha_{1}$, the axial force only shifts $\omega_{1}$ and the pull-in instability when varying from compressive to tensile loading. The fundamental frequency $\omega_{1}$ behavior is a softening behavior with DC as shown in Figure 4a. At low DC voltages, there is no tunability in frequency for medium and high values of $\alpha_{1}$ when there is tensile stress shown in figures $4 \mathrm{~b}$ and 4c. However, the fundamental frequency of the microbeam resonator switches from small softening behavior to hardening throughout the range of DC voltage for compressive axial load as well as no stress conditions. At high values of DC voltages, the microbeam resonator is highly tunable for medium and high $\alpha_{1}$. Though, the resonator is highly unstable for medium values $\alpha_{1}$. These shifts in $\omega_{1}$ and pull-in instability proved in [19] for the same values of DC voltages and for low values of $\alpha_{1}$. 

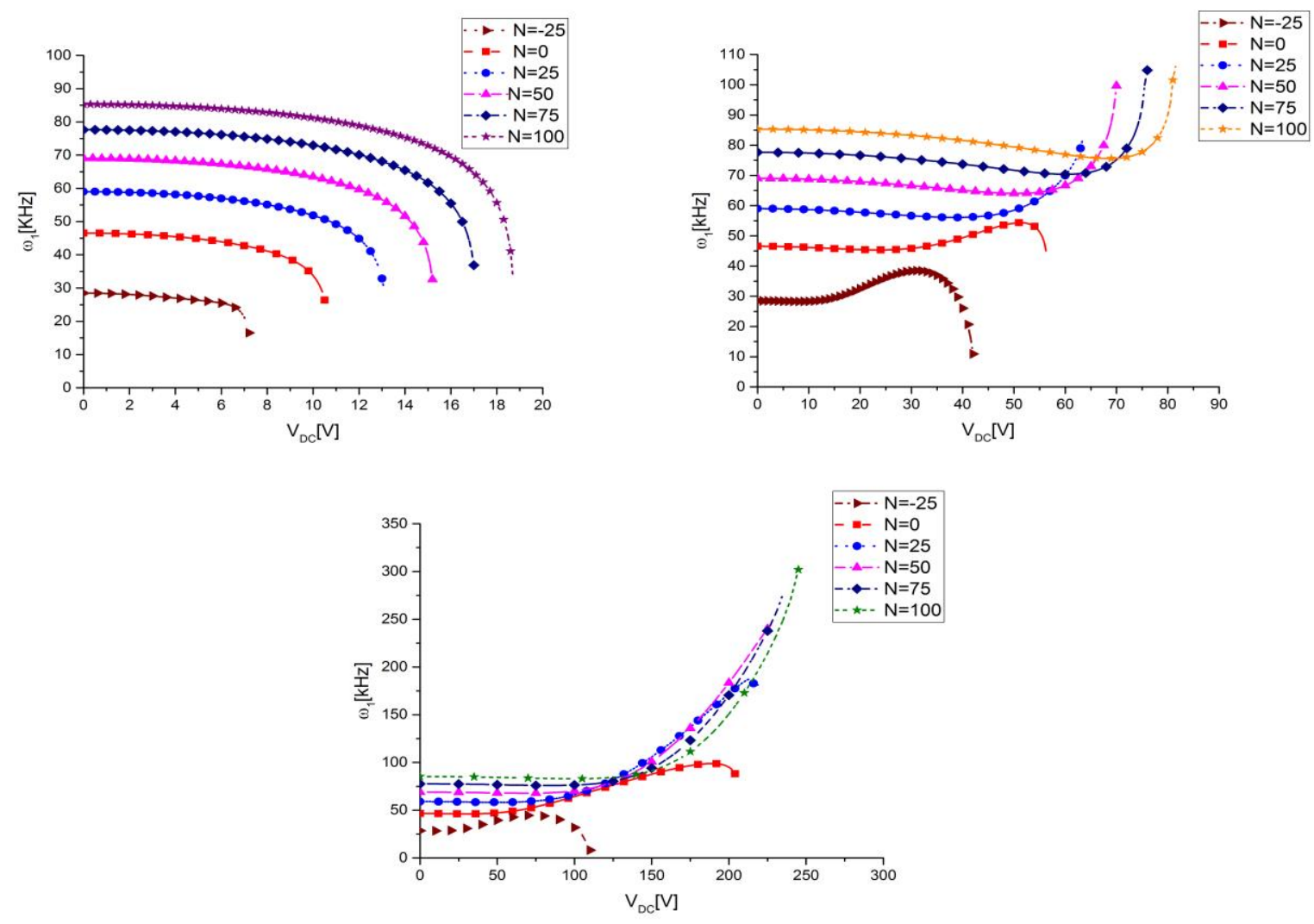

Figure 4: Fundamental natural frequency versus DC voltage for different values of axial load $N$ for $\alpha_{1}=6$; (a); $\alpha_{1}=37.5$; (b); and $\alpha_{1}=121.5$; (c).

To further understand the frequency shift due to the DC voltage under various $\alpha_{1}$, we solve equation (16) employing one symmetric mode in the Galerkin discretization and assuming a harmonic motion in the first mode shape $u_{1}(t)=e^{j{ }^{t}}$. Then equation (16) can be written as

$$
\omega_{1}^{2}+\omega_{n o n, 1}^{2}=\left[2 \alpha_{1} \int_{0}^{1} \phi_{1} \frac{\partial^{2} w_{s}}{\partial x^{2}} d x \int_{0}^{1} \phi_{1}{ }^{\prime} \frac{\partial w_{s}}{\partial x} d x\right]+\left[\alpha_{1} \int_{0}^{1}\left(\frac{\partial w_{s}}{\partial x}\right)^{2} d x\right] \int_{0}^{1} \phi_{1} \phi_{1}{ }^{\prime} d x+\int_{0}^{1} \frac{2 \alpha_{2} V_{D C}{ }^{2}}{\left(1-w_{s}\right)^{3}} \phi_{1}^{2} d x
$$

The new fundamental frequency under the DC loading can be written as follows:

$$
\omega_{1}^{2}=\omega_{n o n, 1}^{2}+\text { Shift }
$$

Where:

$$
\text { Shift }=\left[-\alpha_{1}\left[2 \int_{0}^{1} \phi_{1} \frac{\partial^{2} w_{s}}{\partial x^{2}} d x \int_{0}^{1} \phi_{1}{ }^{\prime} \frac{\partial w_{s}}{\partial x} d x+\int_{0}^{1}\left(\frac{\partial w_{s}}{\partial x}\right)^{2} d x \int_{0}^{1} \phi_{1} \phi_{1}{ }^{\prime} d x\right]\right]+\left[-\int_{0}^{1} \frac{2 \alpha_{2} V_{D C}{ }^{2}}{\left(1-w_{s}\right)^{3}} \phi_{1}{ }^{2} d x\right]
$$

The right hand side of equation (22) is composed of the summation of the shift due to the mid-plane stretching and the shift due to the electrostatic force, respectively. We show in figure 5a the nondimensional shift of the natural frequency, Shift, as a function of the DC voltage for the same beam described in Section 3 for a fixed $d=7 \mu \mathrm{m}$. As shown in figure $5 \mathrm{~b}$, the shift due to the electrostatic force is always a negative quantity, thus leading as expected to the softening effect. Hence, it acts to reduce the natural frequency of the beam since and decreases its stiffness. On the other hand, the shift due to midplane stretching, figure $5 \mathrm{c}$ is always a positive quantity, which tends to raise the natural frequency. Adding both opposing effects leads to the total shift in figure $4 \mathrm{a}$, in which case the effect of mid plane 
stretching due to the deformation of the beam from the DC bias happens to overcome the softening effect of the DC bias.

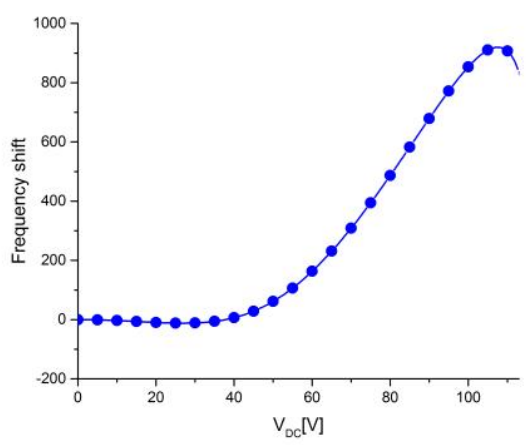

(a)

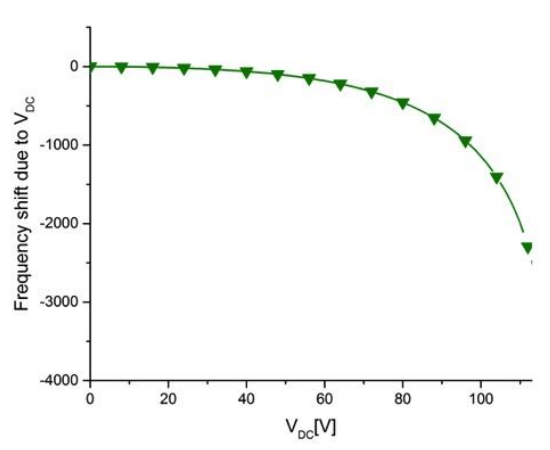

(b)

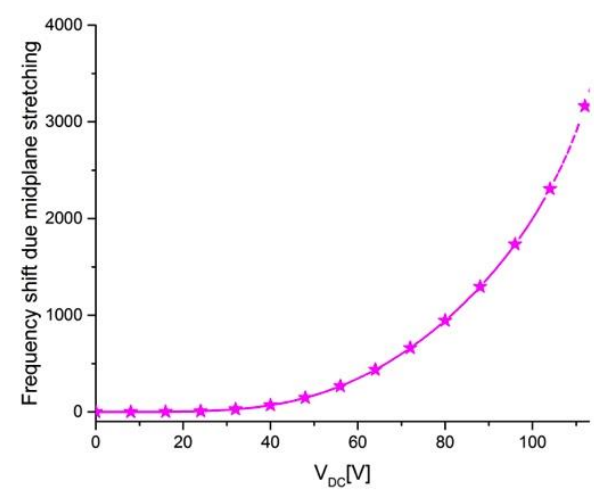

(c)

Figure 5: Frequency shift versus DC polarization voltage for $\alpha_{1}=62.5$ (a) total shift, (b) shift due to electrostatic force, and (c) shift due to mid-plane stretching.

Next, we study the effect of the electrostatic force as well as the ratio between the air gap and the thickness of the microbeam on the higher modes of vibrations. Figure 6 shows the variation of the second and third symmetric dimensional natural frequencies as a function of the DC loading for different values of $\alpha_{1}$ for the parameters of Table 1 . We demonstrate that the effect of the electrostatic force is not strong for higher order modes. The shift is more influenced by the mid-plane stretching due to the static deformation of the beam from the DC bias that leads to an increase of the second and third symmetric natural frequencies as increasing the DC load. 


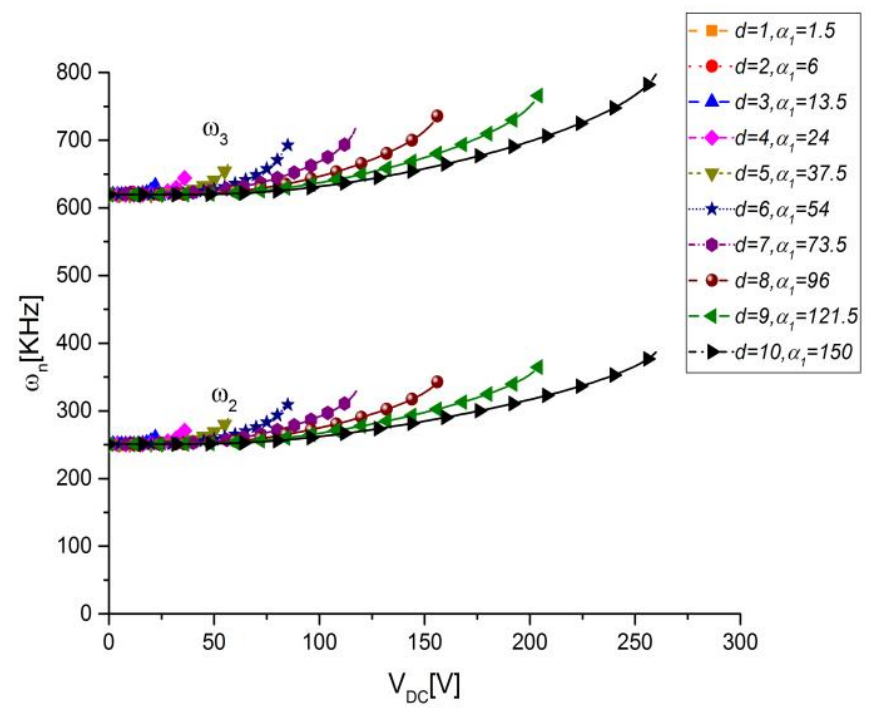

Figure 6: Second and Third symmetric dimensional natural frequencies versus the DC polarization voltage for different values of $\alpha_{1}$.

\section{Experimental}

Setup

We conducted our experimental investigation on a polysilicon chip containing clamped-clamped straight microbeams with different $l, h$, and $d$ dimensions. These microbeams were fabricated from SOI wafers with highly conductive $25 \mu \mathrm{m}$ Si device layer by MEMSCAP [27]. For dynamic measurements and to perform frequency sweeps, we used a Micro System Analyzer with in-plane microstructure vibration and motion analysis using stroboscopic video microscopy from Polytec [28] shown in figure 7a. At the beginning, we measure the linear resonance frequency using FFT to identify its location. Then, we perform and show forward frequency response sweeps using electrostatic forcing around the fundamental natural frequency $\omega_{l}$ while maintaining small $\mathrm{AC}$ voltage values to maintain linear behavior response. In our experiment, two microbeams are considered that are different in the gap $d$ between the microbeam and stationary electrodes. Their length and width dimensions are $600 \mu \mathrm{m}$ and $25 \mu \mathrm{m}$, respectively. Their gaps separating them from the stationary electrodes have nominal values of $7 \mu \mathrm{m}$ and $8 \mu \mathrm{m}$. A top view of one of the beams is shown in figure $7 b$. 


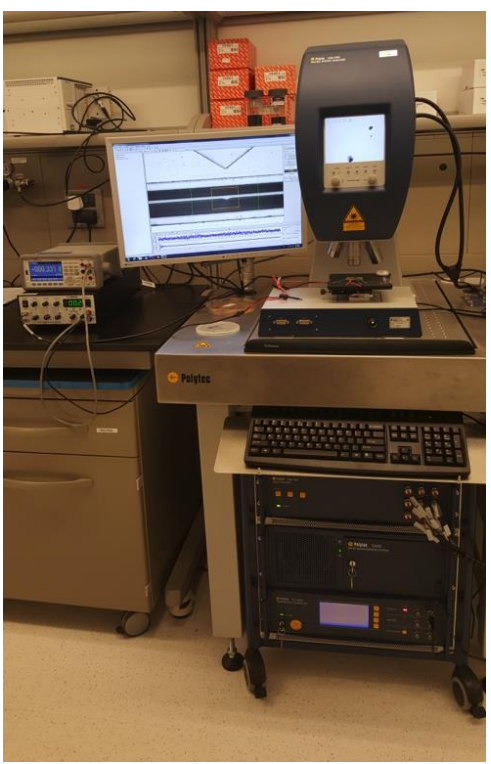

(a)

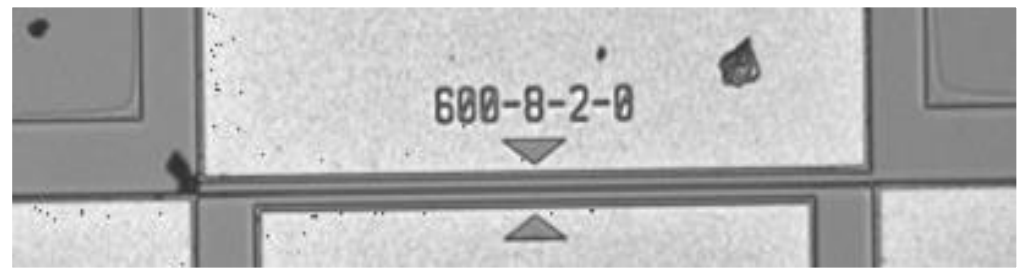

(b)

Figure 7: (a) The experimental set-up. (b) Top view of a microbeam of $l=600 \mu \mathrm{m}, b=25 \mu \mathrm{m}, h=2 \mu \mathrm{m}$ and $d=8 \mu m$.

\section{Comparison of experimental data with the theoretical results}

In this section, we aim to demonstrate experimentally the increase of the natural frequency of the microbeam as increasing the DC voltage for high values of $\alpha_{1}$. To investigate the resonance frequency of the studied beams, we proceed by conducting frequency sweep tests for different DC voltages and $V_{A C}=3 \mathrm{~V}$ as shown in figures $8 \mathrm{~b}$ and $8 \mathrm{~d}$. To guarantee linear frequency-response curves, and hence avoid any softening or hardening behavior in these curves, we limit the $\mathrm{DC}$ voltage by $V_{D C}=90 \mathrm{~V}$ for the beam with the nominal gap $7 \mu \mathrm{m}$ (beam 1 ) and $V_{D C}=95 \mathrm{~V}$ for the beam with nominal gap $8 \mu \mathrm{m}$ (beam 2).

Figures 8a and 8c show that for the small DC loads, the natural frequency of the beam decreases as demonstrated in Section 4. Increasing the DC voltage further leads to the raise of the natural frequency as well. We reach for beam 1 a raise of $25 \%$ and for beam 2 a raise of $35 \%$ of the natural frequency. We found that the thickness of the beam is not uniform along the beam. Hence, in our simulation we try to estimate an effective $\alpha_{1}$, which takes into consideration the non-uniformity of the thickness. For beam 1 , we found that the effective $\alpha_{l}=38$ and for beam 2 the effective $\alpha_{l}=60$. The figures show good agreement between the experimental data and the analytical results. 


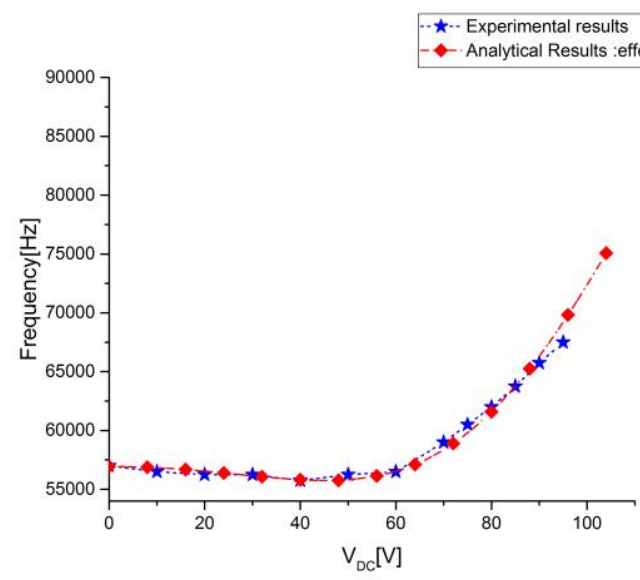

(a)

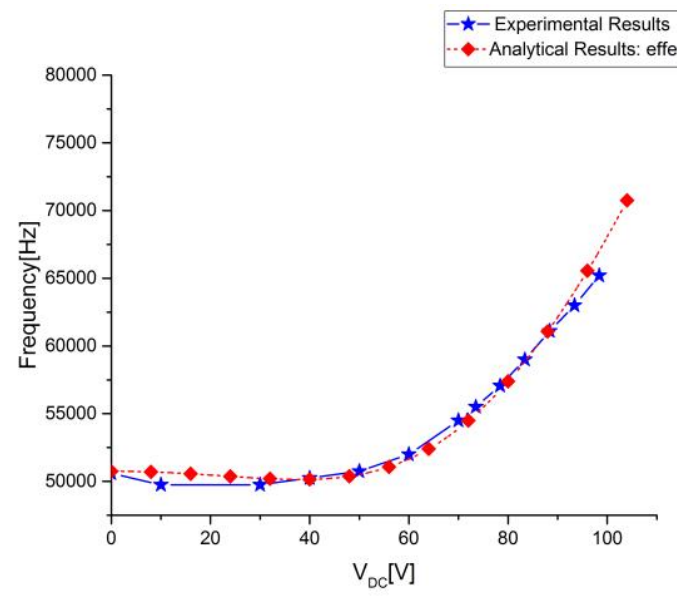

(c)

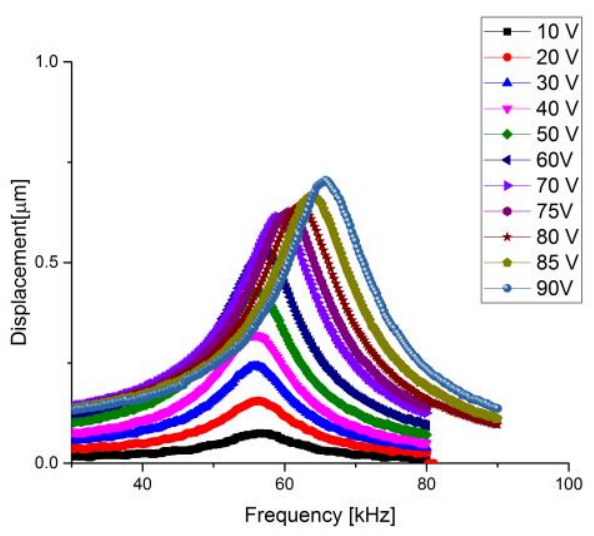

(c)

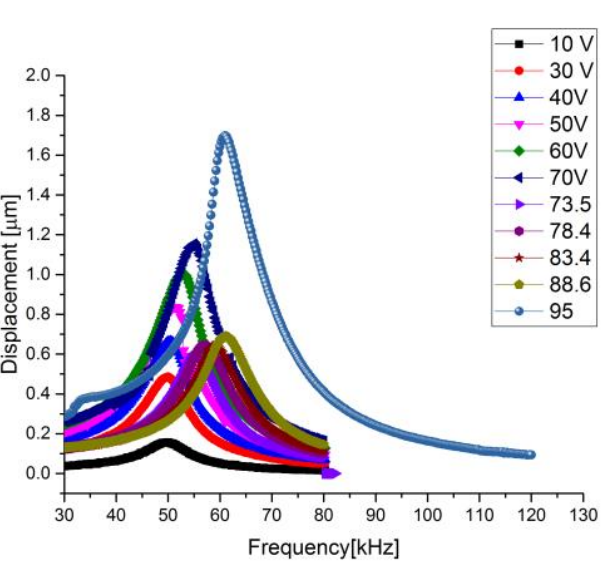

(d)

Figure 8: First natural frequency versus DC polarization voltage and the frequency response curve of different DC voltage with $V_{A C}=3 \mathrm{~V}$ respectively for beam 1 (a) and (b) and beam 2 (c) and (d).

\section{Conclusions}

We studied the combined effect of the mid-plane stretching and the electrostatic force on the variation of the natural frequencies of the electrostatically actuated microbeams. We presented the adopted analytical model describing the system and compared them to the experimental data carried out for two beams. A good agreement was shown between both results. We calculated the static deflection of the microbeam as a function of DC voltage for different values of $\alpha_{1}$. We found that the maximum deflection decreases for high values of $\alpha_{1}$ as the same level of DC voltage and the pull-in instability is shifted for higher values of DC voltage since the stiffness of the beam increases. Increasing $\alpha_{1}$ was found to change the behavior of the variation of the natural frequency of the microbeam. We found that for low values of $\alpha_{1}$, the effect of mid-plane stretching is negligible compared to the electrostatic force and then the fundamental natural frequency decreases as increasing the DC voltage. For high values of $\alpha_{1}$, the effect of mid-plane stretching due to the deformation of the beam under the DC load overpasses the effect of the electrostatic force and then the natural frequency is shifted for higher values as increasing the DC voltage, which can lead to a raise of more than $100 \%$. Also, we studied the effect of axial stress with the effect of 
DC voltage for different scales of $\alpha_{1}$. We found that the microbeam resonator is highly tunable for medium and high $\alpha_{1}$ at high values of DC voltages but it is highly unstable for medium scales of $\alpha_{1}$. At compressive and no stress conditions, the fundamental frequency of the microbeam resonator switches from small softening behavior to hardening throughout the range of DC voltage for medium and high scales of $\alpha_{1}$.

We demonstrated these results experimentally for two microbeams with two different values of $\alpha_{1}$ and we found a good agreement between the experimental and the analytical results. We demonstrated that the effect of the electrostatic force is not strong on the higher order modes when influenced by the mid-plane stretching due to the static deformation of the beam from the DC bias. In conclusion, we demonstrated that one can effectively tune the frequency of electrostatically actuated resonator by varying the DC polarization voltage and by controlling the ratio between the air gap and the thickness of the microbeam.

\section{References:}

[1] Girbau D, Lázaro A, Pérez A, Martínez E, Pradell L, Villarino R. Tunable dual-band resonators for communication systems. International Journal of Microwave and Wireless Technologies. 2010;2(3-4):245.

[2] Sirci S, Martinez J, Taroncher M, Boria V, editors. Varactor-loaded continuously tunable SIW resonator for reconfigurable filter design. Microwave Conference (EuMC), 2011 41st European; 2011: IEEE.

[3] Sonmezoglu S, Alper SE, Akin T. An automatically mode-matched MEMS gyroscope with wide and tunable bandwidth. Microelectromechanical Systems, Journal of. 2014;23(2):284-97.

[4] Peters C, Maurath D, Schock W, Mezger F, Manoli Y. A closed-loop wide-range tunable mechanical resonator for energy harvesting systems. Journal of Micromechanics and Microengineering. 2009;19(9):094004.

[5] Zhuang L, Beeker W, Leinse A, Heideman R, Roeloffzen C, editors. Continuously tunable photonic fractional Hilbert transformer using ring resonators for on-chip microwave photonic signal processing. Microwave Photonics (MWP), 2012 International Topical Meeting on; 2012: IEEE.

[6] Nayfeh AH, Younis MI, Abdel-Rahman EM. Reduced-order models for MEMS applications. Nonlinear dynamics. 2005;41(1-3):211-36.

[7] Sazonova V, Yaish Y, Üstünel H, Roundy D, Arias TA, McEuen PL. A tunable carbon nanotube electromechanical oscillator. Nature. 2004;431(7006):284-7.

[8] Ouakad HM, Younis MI. Natural frequencies and mode shapes of initially curved carbon nanotube resonators under electric excitation. Journal of Sound and Vibration. 2011;330(13):3182-95.

[9] Tajaddodianfar F, Pishkenari HN, Yazdi MRH, Miandoab EM. Size-dependent bistability of an electrostatically actuated arch NEMS based on stain gradient theory. Journal of Physics D: Applied Physics. 2015;48(24):245503.

[10] Kozinsky I, Postma HC, Bargatin I, Roukes M. Tuning nonlinearity, dynamic range, and frequency of nanomechanical resonators. Applied Physics Letters. 2006;88(25):253101.

[11] Akgöz B, Civalek Ö. Buckling analysis of functionally graded microbeams based on the strain gradient theory. Acta Mechanica. 2013;224(9):2185-201.

[12] Miandoab EM, Yousefi-Koma A, Pishkenari HN. Nonlocal and strain gradient based model for electrostatically actuated silicon nano-beams. Microsystem Technologies. 2014;21(2):457-64.

[13] Remtema T, Lin L. Active frequency tuning for micro resonators by localized thermal stressing effects. Sensors and Actuators A: Physical. 2001;91(3):326-32.

[14] Yamamoto T, Inomata K, Watanabe M, Matsuba K, Miyazoki T, Oliver W, Nakamura Y and Tsai J S 2008 Flux-driven Josephson parametric amplifier Appl. Phys. Lett. 93042510.

[15] Nathanson HC, Newell WE, Wickstrom R, Davis Jr JR. The resonant gate transistor. Electron Devices, IEEE Transactions on. 1967;14(3):117-33.

[16] Krylov S, Ilic BR, Schreiber D, Seretensky S, Craighead H. The pull-in behavior of electrostatically actuated bistable microstructures. Journal of Micromechanics and Microengineering. 2008;18(5):055026.

[17] Tilmans HA, Legtenberg R. Electrostatically driven vacuum-encapsulated polysilicon resonators: Part II. Theory and performance. Sensors and Actuators A: Physical. 1994;45(1):67-84.

[18] Batra R, Porfiri M, Spinello D. Vibrations of narrow microbeams predeformed by an electric field. Journal of Sound and Vibration. 2008;309(3):600-12. 
[19] Abdel-Rahman EM, Younis MI, Nayfeh AH. Characterization of the mechanical behavior of an electrically actuated microbeam. Journal of Micromechanics and Microengineering. 2002;12(6):759.

[20] Najar F, Abdel-Rahman E, Nayfeh A, Choura S, editors. Analysis of the Orbits of Electrostatic MEMS Resonators. ASME 2008 International Mechanical Engineering Congress and Exposition; 2008: American Society of Mechanical Engineers.

[21] Younis M, Abdel-Rahman EM, Nayfeh A. A reduced-order model for electrically actuated microbeambased MEMS. Microelectromechanical Systems, Journal of. 2003;12(5):672-80.

[22] Ahn Y, Guckel H, Zook JD. Capacitive microbeam resonator design. Journal of Micromechanics and Microengineering. 2001;11(1):70.

[23] Kuang J-H, Chen C-J. Dynamic characteristics of shaped micro-actuators solved using the differential quadrature method. Journal of Micromechanics and Microengineering. 2004;14(4):647.

[24] Najar F, Choura S, El-Borgi S, Abdel-Rahman E, Nayfeh A. Modeling and design of variable-geometry electrostatic microactuators. Journal of Micromechanics and Microengineering. 2005;15(3):419.

[25] Hung ES, Senturia SD. Extending the travel range of analog-tuned electrostatic actuators. Microelectromechanical Systems, Journal of. 1999;8(4):497-505.

[26] Younis MI. MEMS linear and nonlinear statics and dynamics: Springer Science \& Business Media; 2011.

[27] http://www.memscap.com/

[28] http://www.polytec.com/us/ 